\title{
Altos custos financeiros do trauma vascular
}

\section{Expensive therapy of vascular trauma}

\author{
Ricardo Costa-Val, TCBC-MG'; Maria Cristina Marques²
}

\author{
RES U M O
}

\begin{abstract}
Objetivo: Demonstrar o custo e impacto financeiro referente à primeira abordagem cirúrgica das lesões vasculares em pacientes admitidos no Hospital João XXIII/FHEMIG, entre os anos de 2004 a 2006. Métodos: Trata-se de um estudo com aprovação ética, retrospectivo, de coorte e descritivo realizado a partir da auditoria de contas hospitalares referentes a 70 prontuários catalogados pelo Serviço de Trauma Cardiovascular. Resultados: Cinco (7,14\%) prontuários foram excluídos por má qualidade técnica. 0 valor monetário repassado pelo Sistema Único de Saúde e pelo setor privado foram de R\$103.614,96 (US\$60.949,97) e de R\$185.888,21 (US\$ 109.346,0, respectivamente, implicando em defasagem potencial de 44\%. Houve correlação direta entre custos e topografia anatômica das lesões e exponencial em relação às variáveis hemoderivados e próteses vasculares. Conclusão: Este estudo corrobora os altos custos do trauma vascular e fortalece a importância da auditoria de contas para as tomadas de decisões médicas.
\end{abstract}

Descritores: Ferimentos e lesões. Tecnologia de alto custo. Auditoria financeira.

\section{INTRODUÇÃO}

O trauma é um dos maiores desafios da era moderna para as práticas de saúde devido às altas taxas de morbi-mortalidade e por seu expressivo custo financeiro, particularmente nos países em desenvolvimento, como o Brasil. Apenas no ano de 2003 foram internadas em nosso país 753.304 vítimas de trauma nos hospitais conveniados ao Sistema Único de Saúde (SUS) e cujo custo financeiro hospitalar despendido foi $27 \%$ maior do que todas as outras causas de internação, excetuando às relacionadas com os partos ${ }^{1-2}$.

Avaliar o trauma vascular, seja na zona rural ou nos grandes centros urbanos, não é tarefa simples já que envolve inúmeros fatores, tais como ausência de padronização para a coleta dos dados, questões sociais correlacionadas e as sub-notificações ${ }^{2-5}$.

Reconhece-se cada vez mais a relevância da auditoria de contas para as instituições hospitalares e gestores de saúde devido o fato da mesma possibilitar a análise técnica e a identificação de situações administrativas inadequadas. Por meio da análise pericial dos procedimentos hospitalares é possível também a avaliação sistemática da qualidade da assistência à saúde, além de se aumentar a possibilidade do ressarcimento despesas dos materiais e procedimentos empregados. Ademais, não é incomum o desperdício e perdas desnecessárias em consequência do mau planejamento ou pela utilização indevida de materiais, equipamentos e dos recursos huma$\operatorname{nos}^{5-9}$.

O objetivo deste trabalho é demonstrar o custo financeiro e impacto da primeira abordagem cirúrgica das lesões vasculares em pacientes admitidos no Hospital João XXIII/FHEMIG, entre os anos de 2004 a 2006 e ainda suas implicações para as tomadas de decisões médicas.

\section{MÉTODOS}

Trata-se de um estudo retrospectivo de coorte e descritivo aprovado pelo Comitê de Ética em Pesquisa da FHEMIG/CONEP, registro 575/2007, feito a partir da análise das contas hospitalares de todos os prontuários elegíveis catalogados pelo Serviço de Trauma Cardiovascular.

Foram considerados como critérios de inclusão a coerência entre os diagnósticos, os códigos de procedimentos - SUS e os materiais exclusivamente utilizados dentro do centro cirúrgico durante a primeira abordagem operatória.

Os critérios de exclusão foram: 1) prontuários preenchidos de forma incorreta e/ou dúbia; 2) prontuários pertencentes a pacientes que tinham lesão isolada de artéria e/ou veia de médio e pequeno calibre, definindo-se previamente que enquadravam nestas condições artéria e/ ou veia radial, artéria e/ou veia ulnar, vasos dos quirodáctilos, artéria dorsal anterior, vasos dos pododáctilos,

Trabalho realizado no Serviço de Trauma Cardiovascular do Hospital João XXIII / FHEMIG.

1. Titular da Cirurgia do Trauma - FHEMIG - Belo Horizonte - MG-BR; 2. Enfermeira Especializada em Auditória em Sistemas de Saúde - FHEMIG - Belo Horizonte - MG-BR. 
artéria temporal, veia jugular externa, veia cefálica e/ou basílica, veia safena magna e/ou safena parva bem como suas tributárias e vasos intra-abdominais de menor porte.

A estimativa do custo, em reais, levou em consideração todos os materiais médico-hospitalares, incluindo medicamentos, órteses e prótese, gases medicinais e hemoderivados utilizados. Estratificaram-se ainda, os resultados por topografias anatômicas das lesões e por clínicas cirúrgicas. As lesões cardíacas foram incluídas dentro do grupo de lesões vasculares.

Por outro lado, não foram computados os custos relacionados a depreciações de equipamentos, uso de sala cirúrgica, internação e remunerações aos profissionais da saúde. Utilizou-se a relação de US\$1 = R\$ 1,70 (abril/08).

O repasse financeiro teve como parâmetro os valores referentes aos códigos de procedimentos do Sistema Único de Saúde - SUS/2007 e a Planilha de Registro de Preços de Material Médico-Hospitalar da FHEMIG/2006.

Já a projeção para o setor privado se baseou no Índice Referencial de Medicamentos - Brasíndice/2007 e no Índice de Material Médico Hospitalar da União Nacional das Instituições de Auto Gestão em Saúde - Unidas/ 2006.

\section{RESULTADOS}

Foram avaliados 70 prontuários e destes excluídos cinco $(7,14 \%)$ em virtude da má qualidade técnica dos registros. Nos demais houve lesões vasculares em 34 $(52,3 \%)$ e lesões vasculares associadas em 31 (47,7\%) casos, subdivididas nas seguintes grandes áreas: cirurgia geral $(41,9 \%)$, ortopedia (35,5\%), cirurgia plástica $(19,4 \%)$ e neurocirurgia (3,2\%).

O valor do repasse feito pelo SUS foi de $R \$$ 103.614,96 (mediana de $R \$ 1.432,88 ; \pm R \$ 909,8$ ) e o custo financeiro estimado para o setor privado de saúde foi de $R \$ 185.888,21$ (mediana de $R \$ 2.236,55 ; \pm R \$ 1.546,34$ ), projetando defasagem de $44,3 \%$. No entanto não houve homogeneidade para ambas variáveis (Figuras 1 e 2).

A estimativa dos custos para o sistema privado e o repasse financeiro do SUS com seus impactos correlacionados para apenas os casos com lesões vasculares foram, respectivamente, $\mathrm{R} \$ 96.171,32$ (impacto de 52\%) e $\mathrm{R} \$ 42.904,27$ (impacto de 41\%).

No entanto, a associação com lesões não vasculares amenizou os custos financeiros como se pode perceber pela redução de seu respectivo impacto para a medicina privada - custos de $\mathrm{R} \$ 89.716,89$ e impacto de $48 \%$ e de fato, provocou significativo ganho em relação ao repasse do SUS - R\$ 60.710, 69 e impacto de 59\%.

O emprego de hemoderivados e de próteses vasculares aumentou os custos financeiros de maneira diretamente proporcional quando se utilizou apenas destes itens e de maneira exponencial para a utilização simultânea de ambos. Contudo, a associação entre lesões

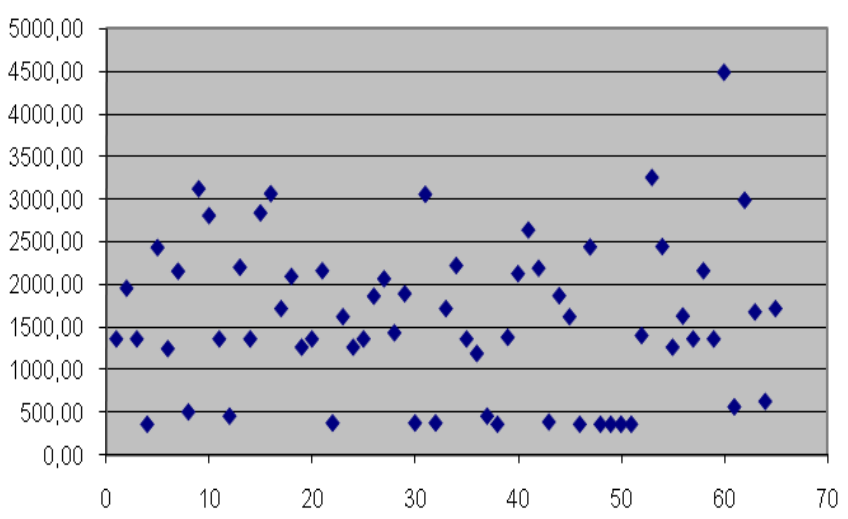

Figura 1 - Dispersão dos valores repassados pelo SUS, em reais, para todos os casos.

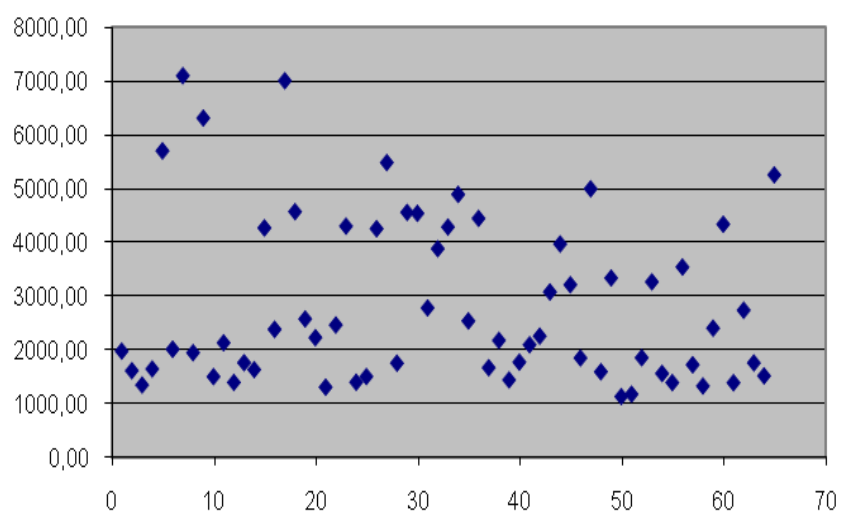

Figura 2 - Dispersão dos valores referentes ao custo operatório total, em reais, projetado para a medicina privada, segundo Brasíndice e Unidas.

vasculares e não vasculares minimizou o impacto financeiro (Tabelas 1 a 3).

Por outro lado, a utilização do cateter de Fogarty ${ }^{\circledR}$ não influenciou os custos financeiros, seja nos casos com lesões vasculares isoladas ou não ou ainda utilização de hemoderivados e/ou próteses vasculares (15 casos: custo estimado de $\mathrm{R} \$ 40.762,99$ com impacto de $22 \%$ para setor privado e repasse pelo SUS de R $\$ 30.799,44$ com de $30 \%$ impacto).

E finalmente, a correlação com topografia anatômica das lesões vasculares e as variáveis hemoderivados e próteses vasculares causou aumento dos custos financeiros estimados para a assistência médica privada e sobre o repasse do SUS da seguinte maneira: 1) lesões abdominais e de membros inferiores: 38 casos (58\%), estimativa dos custos de $\mathrm{R} \$ 109.734,72$; impacto de $59 \%$, repasse público de $\mathrm{R} \$ 62.654,43$, impacto de $60,5 \%$; 2) lesões nos membros superiores: 16 casos (25\%), estimativa dos custos de $\mathrm{R} \$ 27.784,90$, impacto de $15 \%$, repasse público de $\mathrm{R} \$ 24.343,96$, impacto de $23,5 \%$; 3 ) lesões cervicotorácicas: 11 casos (17\%), estimativa dos custos de $R \$ 48.368,59$, impacto de $26 \%$, repasse público de $R \$ 16.616,57$, impacto de $16 \%$. 
Tabela 1 - Projeção comparativa do custo operatório total e seu impacto segundo o Brasíndice e Unidas, em reais, nos casos com lesões vasculares que utilizaram apenas hemoderivados.

\begin{tabular}{lccc}
\hline Caso & Custo Total & Repasse SUS & \% do repasse \\
\hline 22 & 2472,76 & 373,58 & $15,11 \%$ \\
$30 *$ & 4548,91 & 373,58 & $8,21 \%$ \\
$32 * *$ & 3890,74 & 373,58 & $9,60 \%$ \\
38 & 2185,10 & 358,58 & $16,41 \%$ \\
46 & 1858,59 & 358,58 & $19,29 \%$ \\
49 & $3.345,75$ & 358,58 & $10,72 \%$ \\
Total & $18.301,85$ & $2.196,48$ & Mediana $13 \%$ \\
Impacto nos custos (\%) & $9,8 \%$ & $2,1 \%$ & \\
\hline
\end{tabular}

* Utilizou papa de hemácias e plasma fresco.

* Utilizou papa de hemácias, plasma fresco e concentrado de plaquetas.

Tabela 2 - Projeção comparativa dos custos operatórios e seu impacto segundo o Brasíndice e Unidas, em reais, dos casos com lesões associadas que utilizaram apenas hemoderivados.

\begin{tabular}{lccc}
\hline Caso & Custo Total & Repasse SUS & \% do repasse \\
\hline 36 & 4455,32 & 1190,65 & $26,72 \%$ \\
43 & 3086,64 & 386,06 & $12,51 \%$ \\
$56^{*}$ & 3549,68 & 1630,88 & $45,94 \%$ \\
Total & $11.091,64$ & $3.207,59$ & Mediana $27 \%$ \\
Impacto nos custos (\%) & $5,1 \%$ & $3,1 \%$ & \\
\hline
\end{tabular}

*Utilizou papa de hemácias e plasma fresco.

Tabela 3 - Projeção comparativa do custo operatório total e seu impacto, em reais, para todos os casos que utilizaram hemoderivados e prótese vascular de PTFE ${ }^{\circledR}$.

\begin{tabular}{|c|c|c|c|}
\hline Caso & Custo Total & Repasse SUS & $\%$ do repasse \\
\hline 5 & 5712,13 & 2435,26 & $42,63 \%$ \\
\hline 7 & 7108,10 & 2157,06 & $30,35 \%$ \\
\hline 17 & 7015,63 & 1718,28 & $24,49 \%$ \\
\hline 23 * & 4310,38 & 1622,08 & $37,63 \%$ \\
\hline 26 * & 4263,03 & 1863,40 & $43,71 \%$ \\
\hline 27 * & 5493,32 & 2068,00 & $37,65 \%$ \\
\hline 29 & 4566,83 & 1893,03 & $41,45 \%$ \\
\hline 33 * & 4293,51 & 1718,28 & $40,02 \%$ \\
\hline 34 * & 4901,53 & 2223,68 & $45,37 \%$ \\
\hline 45 & 3221,43 & 1622,08 & $50,35 \%$ \\
\hline 60 & 4345,67 & 4494,55 & $103,43 \%$ \\
\hline $65 *$ & 5264,85 & 1718,28 & $32,64 \%$ \\
\hline Total & $60.496,41$ & $25.333,98$ & Mediana 41\% \\
\hline Impacto nos custos (\%) & $33 \%$ & $25 \%$ & \\
\hline
\end{tabular}

*Somente casos da CCV.

\section{DISCUSSÃO}

A casuística empregada, que representou $2 / 3$ de todos os casos registrados no período, as recentes versões de tabelas do SUS, Brasíndice e Unidas, a análise dos materiais empregados somente no centro cirúrgico e ainda a exclusão das despesas indiretas, entendidas como o uso ou a depreciação de materiais permanentes, minimizou vários potenciais vieses.

A pequena diferença entre os valores dos códigos de procedimentos lançados no espelho da conta e a tabela dos códigos por procedimentos pagos pelo SUS foi devida às taxas de longa permanência contemplada no SH acrescida em $50 \%$ por se tratar de um centro de referên- 
cia para atendimentos de urgências e emergências médicas e que é denominado pelo SUS de Índice de Valorização Hospitalar $2,6,7$.

A distribuição heterogênea dos custos financeiros foi devida a não uniformidade das lesões vasculares e não vasculares, apesar das mesmas ocorrerem em topografias anatômicas frequentemente citadas em vítimas de trauma. Na verdade, esta é mais uma característica do trauma, desafiante agravo a saúde e que se revela devastador em todas as regiões do corpo humano 1;3;5;-11.

Sem sombras de dúvidas, o trauma representa ainda um dos maiores consumidores dos recursos financeiros despendidos na assistência à saúde, sejam diretos ou relacionados à perda da capacidade produtiva, dados observados e que corroboram a literatura científica ${ }^{4-15}$

Analisar todos os aspectos relacionados ao trauma e suas variantes, como o trauma vascular, rotineiramente tem sido progressivamente destacado como não apenas importante, mas essencial para a própria melhoria da qualidade assistencial e administrativa ${ }^{2,4,5,12-18}$.

Dentro do contexto das boas práticas institucionais a auditoria se revela importante ferramenta para o Sistema Integrado de Gestão Hospitalar já que é parte desta a análise dos espelhos de conta de cada prontuário, o que possibilita entender seus detalhes, tais como os Serviços Hospitalares (SH), Serviços Profissionais (SP), órteses e próteses (OPME) e os Serviços Auxiliares de Diagnóstico e Terapêutico (SADT) 6-8,15.

Bom exemplo da relevância de práticas administrativas capazes de minimizar os custos e impacto financeiro da abordagem do trauma vascular no setor privado se faz pelo próprio "pregão eletrônico", responsável pela redução média de $20 \%$ para os medicamentos e de 10 a $42 \%$ para as órteses/próteses quando se tem como base as tabelas Brasíndice e das Unidas.

Por outro lado, é preocupante a dificuldade por parte do médico em entender e registrar adequadamente seus atos profissionais, o significado dos códigos dos procedimentos. A perpetuação desta postura é um dos fatores responsáveis para a incapacidade que de maneira geral, tem o profissional médico, elemento universal na assistência a saúde, para gerir efetivamente as instituições de saúde. Soma-se a isto a constante e elevada taxa de glosas, mais um fator para reduzir o escasso rendimento deste profissional ${ }^{6-8}$.
Fica evidente então a importância de se fazer entender regras básicas como, por exemplo, o próprio SUS, cujos repassem se dão pela coerência entre o diagnóstico, os atos realizados e respectivos registros, todos estes englobados nos pacotes de procedimentos codificados da seguinte formado por oito dígitos (XX.YYY.ZZ-D) assim designados: $X X$ indica a especialidade, $Y Y Y$ o procedimento, ZZ o órgão ou região anatômica e D o dígito verificador. Por exemplo, o código 48.020.05-2 significa tratamento cirúrgico de lesão vascular traumática de membro inferior unilateral ${ }^{8}$.

A autorização de internação hospitalar (AIH) é o instrumento que deflagra a cobrança da conta hospitalar do paciente e que permite a inclusão de até cinco procedimentos por cada ato operatório realizado a cada 24 horas. Faz-se pertinente explicar dois aspectos que merecem atenção especial, a saber: a necessidade de se conhecer as regras da remuneração pertinentes aos procedimentos de diferentes especialidades médicas realizadas no mesmo ato operatório que comungam das vias de acesso cirúrgico e alguns códigos, sendo o mais comum neste cenário código definido com "politraumatizado" (39.000.00-1). Tais fatos explicam, em parte, a heterogeneidade e dispersão dos valores encontrados, mesmo para casos com os mesmos códigos de procedimentos.

Um sistema de custo com ênfase gerencial e de apoio a gestão dos departamentos produtivos deve informar não apenas quanto custa, mas também por que, para que e como. Faz-se relevante então promover ações institucionais e campanhas esclarecedoras. Os resultados descritos deduzem a real importância de se ter protocolos e padronização de condutas capazes de resultar, conforme a lógica técnica-científica, uso judicioso de hemoderivados e próteses vasculares, principalmente para os casos com apenas lesões vasculares ${ }^{6-8,16,18}$.

Enfim, é preciso entender que as tomadas de decisões médicas "ideais" envolvem também os aspectos econômicos e ainda perceber que existe grande diferença entre deixar de fazer um ato médico por motivos financeiros e optar pela terapia mais coerente sob o ponto de vista econômico 6,7,15-18.

Este estudo corrobora os altos custos do trauma vascular e fortalece a importância da auditoria de contas para as tomadas de decisões médicas.

\section{A B $S$ S T R A C T}

Objective: To analyze all expenses paid to the first surgical approached for vascular lesion patients admitted at Hospital João XXIIII FHEMIG, between years 2004 until 2006. Method: It is about an ethical approved study, retrospective and descriptive from audit over 70 eligible patients enrolled by Cardiovascular Service. Results: Five $(7,14 \%)$ patient's files were excluded for bad quality records. The costs were $R \$ 103.614,96$ (US\$ 60.949,97) and $R \$ 185.888,21$ (US\$109.346,0), a 44\% sliding scale for endowment from Brazilian Public System and private medical assistance estimative, respectively. Indeed, the data showed direct relationship between costs and anatomic topographic and exponential increased expenses when blood and/or vascular prosthesis were utilized. Conclusion: Audit in health system is a must to making decision and evidences the expensive therapy for vascular lesions.

Key words: Wounds and injuries. Technology, high-cost. Financial audit. 


\section{REFERENCIAS}

1. Ministério da Saúde. Vigilância Epidemiológica em Acidentes e Violência. Disponível em: http://portal.saude.gov.br/portal/svs/ visualizar_texto.cfm?idtxt=218533. Acessado em 17 de novembro de 2006 .

2. DATA-SUS. http://w3.datasus.gov.br/datasus. Acessado em 20 de março de 2008.

3. Rich NM. Historical and military aspects of vascular trauma. In: Rich NM, Mattox KL, Hishber GA. Vascular trauma. $2^{\text {nd }}$ ed. Philadelphia: Elsevier Saunders; 2004. p. 3-72.

4. Araújo GR, Mathias SB, Felipe GJ. Dados epidemiológicos. In: Murilo R, Brito CJ, Miguel EV, Miranda FJ, Meirelles SSL. Trauma vascular. Rio de Janeiro: Revinter; 2006. p. 74-82

5. Velinovic MM, Davidovic BL, Lotina IS, Vranes RM, Djukic LP, Arsov JV et al. Complications of operative treatment of injuries of peripheral arteries. Cardiovasc Surg. 2000; 8(4):256-64.

6. Motta ALC. Auditoria de enfermagem nos hospitais e operadoras de planos de saúde. $3^{a}$ ed. São Paulo: látria; 2006.

7. Jacques JE. Gestão hospitalar - Os custos médico-assistênciais. São Leopoldo: Unisinos; 2006.

8. Couto RC, Pedrosa TMG. Hospital - Acreditação e gestão em saúde. Rio de Janeiro: Guanabara Koogan; 2007.

9. Peden M, McGee K, Krug E, editors. Injury: a leading cause of the global burden of disease, 2000. Geneva: World Healthy Organization; 2002.

10. Holcomb JB, Jenkins D, Rhee P, Johannigman J, Mahoney P, Mehta $S$ et al. Damage control resuscitation: directly addressing the early coagulopathy of trauma. J Trauma. 2007; 62(2):307-10.

11. Campos-Christo SF, Miguel EV, Costa-Val R, Teixeira ELC. Amputação primária no trauma: indicações e aspectos legais. In: Murilo R, Brito CJ, Vergara E, Meirelles S. Trauma vascular. Rio de Janeiro: Revinter; 2006. p. 153-60.

12. Yoshida WB. A nossa produção científica invisível [editorial]. J Vasc Bras 2005: 4(2):113
13. Salenius JP, Lepäntalo M, Ylönen K, Luther M. Treatment of peripheral vascular diseases-basic data from the nationwide vascular registry FINNVASC. Ann Chir Gynaecol. 1993; 82(4):23540

14. Magee TR, Collin J, Hands LJ, Gray DW, Roake J. A ten year audit of surgery for vascular trauma in a British teaching hospital. Eur J Vasc Endovasc Surg. 1996; 12(4):424-7.

15. Janne d'Othée B, Langdon DR, Bell GK, Bettmann MA. Operating expenses for the diagnosis and treatment of peripheral vascular disease in an academic interventional radiology department: cost calculations according to a microeconomic method. J Vasc Interv Radiol. 2006; 17(1):85-94

16. Hlaing $T$, Hollister $L$, Aaland $M$. Trauma registry data validation: essential for quality trauma care. J Trauma. 2006; 61(6):1400-7.

17. Guice K, Cassidy L, Mann NC. State Trauma Registries: Survey and Uptade-2004. J Trauma. 2007; 62(2):424-35.

18. Costa-Val R, Campos-Christo SF, Abrantes WL, Campos-Christo MB, Marques MC, Vergara EM. Reflexões sobre o trauma cardiovascular civil a partir de um estudo de 1000 casos atendidos em um centro de trauma nível I. Rev Col Bras Cir. 2008; 35(3):1627

Recebido em 08/05/2009

Aceito para publicação em 10/07/2009

Conflito de inreresse: nenhum

Fonte de financiamento: nenhuma

\section{Como citar esse artigo:}

Costa-Val R, Marques MC. Altos custos financeiros do trauma vascular. Rev Col Bras Cir. [periódico na Internet] 2010; 37(4). Disponível em URL: http://www.scielo.br/rcbc

\section{Endereço para correspondência:}

Ricardo Costa-Val

E-mail: ricardocostaval@hotmail.com 